\title{
Cerebrospinal fluid synaptosomal- associated protein 25 is a key player in synaptic degeneration in mild cognitive impairment and Alzheimer's disease
}

Hua Zhang ${ }^{1}$, Joseph Therriault ${ }^{2}$, Min Su Kang², Kok Pin $\mathrm{Ng}^{3}$, Tharick A. Pascoal ${ }^{2}$, Pedro Rosa-Neto², Serge Gauthier ${ }^{2^{*}}$ and the Alzheimer's Disease Neuroimaging Initiative

\begin{abstract}
Background: There is accumulating evidence that synaptic loss precedes neuronal loss and correlates best with impaired memory formation in Alzheimer's disease (AD). Cerebrospinal fluid (CSF) synaptosomal-associated protein 25 (SNAP-25) is a newly discovered marker indicating synaptic damage. We here test CSF SNAP-25 and SNAP-25/ amyloid- $\beta 42$ (A $\beta 42$ ) ratio as a diagnostic marker for predicting cognitive decline and brain structural change in the Alzheimer's Disease Neuroimaging Initiative (ADNI) database.

Methods: We stratified 139 participants from the ADNI database into cognitively normal $(C N ; n=52)$, stable mild cognitive impairment ( $(\mathrm{MCl} ; n=22)$, progressive $\mathrm{MCl}(\mathrm{pMCl} ; n=47)$, and dementia due to $\mathrm{AD}(n=18)$. Spearman correlation was performed to test the relationships between biomarkers. Overall diagnostic accuracy (area under the curve (AUC)) was obtained from receiver operating curve (ROC) analyses. Cox proportional hazard models tested the effect of CSF SNAP-25 and SNAP-25/Aß42 measures on the conversion from MCI to AD. Relationships between the CSF SNAP-25 levels, SNAP-25/Aß42 ratio, and diagnostic groups were tested with linear regressions. Linear mixed-effects models and linear regression models were used to evaluate CSF SNAP-25 and SNAP-25/Aß42 as predictors of AD features, including cognition measured by the Mini-Mental State Examination (MMSE) and brain structure and white matter hyperintensity $(\mathrm{WMH})$ measured by magnetic resonance imaging (MRI).

Results: CSF SNAP-25 and SNAP-25/Aß42 were increased in patients with $\mathrm{pMCl}$ and $\mathrm{AD}$ compared with CN, and in $\mathrm{pMCl}$ and $\mathrm{AD}$ compared with $\mathrm{sMCl}$. Cognitively normal subjects who progressed to $\mathrm{MCl}$ or $\mathrm{AD}$ during follow-up had increased SNAP-25/Aß42 ratio compared with nonprogressors. CSF SNAP-25, especially SNAP-25/Aß42, offers diagnostic utility for $\mathrm{PMCl}$ and $\mathrm{AD}$. CSF SNAP-25 and SNAP-25/Aß42 significantly predicted conversion from MCI to AD. In addition, elevated SNAP-25/Aß42 ratio was associated with the rate of hippocampal atrophy in $\mathrm{pMCl}$ and the rate of change of cognitive impairment in $\mathrm{CN}$ over the follow-up period.

(Continued on next page)
\end{abstract}

\footnotetext{
* Correspondence: serge.gauthier@mcgill.ca

Data used in preparation of this article were obtained from the Alzheimer's Disease Neuroimaging Initiative (ADNI) database (adni.loni.usc.edu). As such, the investigators within the ADNI contributed to the design and implementation of ADNI and/or provided data but did not participate in analysis or writing of this report. A complete listing of ADNI investigators can be found at: http://adni.loni.usc.edu/wp-content/uploads/how_to_apply/ ADNI_Acknowledgement_List.pdf

${ }^{2}$ The McGill University Research Centre for Studies in Aging, McGill University, Montreal, Canada

Full list of author information is available at the end of the article
}

(c) The Author(s). 2018 Open Access This article is distributed under the terms of the Creative Commons Attribution 4.0 International License (http://creativecommons.org/licenses/by/4.0/), which permits unrestricted use, distribution, and reproduction in any medium, provided you give appropriate credit to the original author(s) and the source, provide a link to the Creative Commons license, and indicate if changes were made. The Creative Commons Public Domain Dedication waiver (http://creativecommons.org/publicdomain/zero/1.0/) applies to the data made available in this article, unless otherwise stated. 
(Continued from previous page)

Conclusions: These data suggest that both CSF SNAP-25 and SNAP-25/A 42 ratio are already increased at the early clinical stage of AD, and indicate the promise of CSF SNAP-25 and SNAP-25/A 42 ratio as diagnostic and prognostic biomarkers for the earliest symptomatic stage of AD.

Keywords: Alzheimer's disease, Amyloid- $\beta$, Synaptic loss, Synaptosomal-associated protein 25

\section{Background}

Alzheimer's disease (AD) is the most prominent cause of dementia in the elderly. AD is characterized by early loss of synapses in specific brain regions, beginning in the hippocampus and spreading to the neocortex and limbic system, eventually leading to memory impairments [15]. The loss of synapses in AD is greater than the loss of neurons in the cortex, indicating that the synaptic damage precedes the loss of neuronal cell bodies [6-9]. Neuropathological studies have revealed that synaptic loss is already evident at the stage of mild cognitive impairment $(\mathrm{MCI})$ of $\mathrm{AD}[10,11]$. In addition, synaptic loss is closely related with the severity of clinical disease [5]. In view of the above reasons, if there is a biomarker that reflects this pathophysiological process, it may be used to study disease mechanisms, improve tools for early diagnosis, predict progression of disease, and monitor the effects of drugs on reducing the rate of synaptic degeneration in clinical trials of disease-modifying therapies for AD [12]. Therefore, biomarkers that can track synaptic dysfunction in AD may prove useful for more accurate disease staging as well as population enrichment of disease-modifying clinical trials [5].

Synaptic damage can be detected at the earliest stages of AD. MCI patients exhibit loss of presynaptic proteins such as synaptophysin and synaptosomal-associated protein 25 (SNAP-25) and postsynaptic markers such as postsynaptic density-95 and Shank 1 [13]. SNAP-25 is a widely distributed membrane-associated protein that is mainly localized in nerve terminals in the brain. Within the nerve terminals, SNAP-25 is involved in the docking and/or fusion of synaptic vesicles to the plasmalemma, a process essential for synaptic vesicular exocytosis [14]. A study has recently reported increased levels of cerebrospinal fluid (CSF) SNAP-25 in AD patients [15].

However, it is still unknown whether CSF SNAP-25 levels increase at the early clinical stage of $\mathrm{AD}$, and whether CSF SNAP-25 is correlated with other core features of AD such as amyloid- $\beta(\mathrm{A} \beta)$ pathology, structural brain changes, and cognitive decline. In the present study, we tested the hypotheses that CSF SNAP-25 levels and SNAP-25/A $\beta 42$ ratio increase at every stage of $\mathrm{AD}$ and improve the diagnostic accuracy for $\mathrm{AD}$ compared with other core biomarkers. We also tested the hypotheses that CSF SNAP-25 levels and SNAP-25/A $\beta 42$ ratio have associations with $A \beta$ pathology and changes in $\mathrm{AD}$ cognition and brain structure, as measured by the Mini-Mental State Examination (MMSE), Alzheimer's Disease Assessment Scale cognitive subscale (ADAS-cog), and magnetic resonance imaging (MRI).

\section{Methods \\ Database description}

Data used in the preparation of this article were obtained from the Alzheimer's Disease Neuroimaging Initiative (ADNI) database. The ADNI was launched in 2003 as a public-private partnership, led by Principal Investigator Michael W. Weiner, MD. The primary goal of ADNI has been to test whether serial MRI, positron emission tomography (PET), biological markers, and clinical and neuropsychological assessments can be combined to measure the progression of $\mathrm{MCI}$ and early AD. Further information can be found at http://www.adni-info.org.

From the dataset, we selected all participants between 55 years and 90 years (inclusive) of age who had completed lumbar puncture, MMSE, ADAS-cog, Clinical Dementia Rating (CDR) scale, and MRI. Selected individuals were classified as cognitively normal $(\mathrm{CN} ; n=52)$, stable mild cognitive impairment (sMCI; $n=22$ ), progressive $\mathrm{MCI}$ (pMCI; $n=47)$, and dementia due to AD $(n=18)$ according to clinical and behavioral measures provided by the ADNI. In a subanalysis, we also describe $19 \mathrm{CN}$ subjects who progressed to $\mathrm{MCI}(n=12)$ or $\mathrm{AD}(n=7)$ during follow-up.

\section{Classification criteria}

The criteria for $\mathrm{CN}$ included an MMSE score ranging between 24 and 30 , and a CDR score of $0[16,17]$. The criteria for MCI included the presence of a subjective memory complaint, with an MMSE score between 24 and 30, a CDR of 0.5, preserved activities of daily living, and an absence of dementia [18]. In addition to the NINCDS/ADRDA criteria for probable AD, AD dementia subjects had MMSE scores between 20 and 26 and a CDR of 0.5 or 1.0 [19]. We defined sMCI as MCI subjects not progressing to $\mathrm{AD}$ during at least 2 years of follow-up and pMCI as MCI subjects progressing to AD at any time during follow-up [12]. We excluded subjects who were diagnosed with MCI at baseline but reverted to $\mathrm{CN}$ during follow-up, as well as subjects who were diagnosed with $\mathrm{AD}$ at baseline but reverted to $\mathrm{MCI}$ during follow-up. (Further information about the inclusion/exclusion criteria may be found at www.adni-info.org, accessed February 2018.) 


\section{Standard protocol approvals and patient consents}

The ADNI study was approved by the Institutional Review Boards of all the participating institutions. Informed written consent was obtained from all subjects at each center.

\section{CSF analyses}

CSF A 342 , total-tau (t-tau), and phosphorylated-tau at threonine 181 (p-tau) were measured using the multiplex xMAP Luminex platform (Luminex Corp., Austin, TX, USA) and Innogenetics INNO-BIA AlzBio3 (Innogenetics, Ghent, Belgium) immunoassay reagents as described previously [20]. Subjects were classified as A $\beta$ positive or negative using a previously established CSF Aß42 cutoff <192 pg/ml [20]. Mouse anti-human SNAP-25 antibodies were used for the development of an Erenna ${ }^{\circ}$ immunoassay assay according to an agreement between Singulex, Inc. (Alameda, CA, USA) and Washington University. A sandwich enzyme-linked immunosorbent assay (ELISA) was developed using the Erenna immunoassay system to measure SNAP-25 in CSF. Prior to the assay, all samples were centrifuged $(11,000 \mathrm{~g} \times 3 \mathrm{~min})$ to remove particulates. Then, $100-\mu \mathrm{L}$ standards or CSF diluted fourfold were combined with $100 \mu \mathrm{L}$ antibody-coated microparticles diluted in Blocker Casein in TBS plus 1\% Tween-20 for measurement of SNAP-25 in CSF. The assay plate was incubated for $2 \mathrm{~h}$ on a plate shaker set to $525 \mathrm{rpm}$. Microparticles were then magnetically separated and washed once using an Agilent (Santa Clara, CA, USA) Bravo Automated Liquid Handling Platform using Singulex wash buffer. Fluorescent dye-labeled detection antibody diluted in Blocker Casein in TBS plus 1\% Tween-20 (20 $\mu \mathrm{L}$ per well) was added and incubated for $1 \mathrm{~h}$. After washing the magnetic microparticles five times, $20 \mu \mathrm{L}$ per well of Singulex elution buffer was added for $10 \mathrm{~min}$ to separate the detection antibody from the microparticles. Eluted antibodies were then transferred with the Bravo instrument to a clean 384-well plate for reading in the Erenna immunoassay system. All of the CSF data used in this study were obtained from the ADNI files "UPENNBIOMK5-8.csv" and "FAGANLAB_07_15_2015.csv" (accessed February 2018). Further details of ADNI methods for CSF acquisition and measurements and quality control procedures can be found at www.adni-info.org.

\section{Cognitive assessment}

To assess the global cognitive performance we used the MMSE and ADAS-cog scores. MMSE and ADAS-cog scores were selected at six time points: baseline, 6 months, 12 months, 18 months, 24 months, 36 months, and 48 months. The data used in this study was obtained from the ADNI files "MMSE.csv" and "ADAS_ADNI1. csv" (accessed February 2018).

\section{Neuroimaging methods}

To investigate neurodegeneration we used the hippocampal and ventricular volumes. The white matter hyperintensity (WMH) volume, a cerebrovascular disease marker, was also obtained. Those data were obtained from the ADNI files "FOXLABBSI_08_04_17.csv", "UCSDVOL.cSV", and "UCD_ADNI1_WMH.csv" (accessed February 2018). All the imaging data were selected at five time points: baseline, 6 months, 12 months, 24 months, and 36 months. The neuroimaging methods used by ADNI have been described previously [21]. Further details for ADNI image acquisition and processing can be found at www.adni-info.org/methods.

\section{Statistical methods}

Analysis of covariance (ANOVA) and chi-square analyses were performed to test for significant differences between groups on baseline demographics. Associations between the CSF SNAP-25 levels and SNAP-25/A 342 ratio and diagnostic groups were tested with multiple-variable linear regression, adjusted for age and gender. To evaluate whether $A \beta$ influenced these associations, we included the interaction between diagnosis and $\mathrm{A} \beta$ positivity as a predictor in the model.

Spearman correlation was used to test associations between SNAP-25 and SNAP-25/Aß42 and other core biomarkers. Overall diagnostic accuracy (the area under the curve (AUC)) was obtained for each biomarker using receiver operating curve (ROC) analyses. The differences between two AUCs derived from all pairs of two different variables were tested using bootstrap methods.

The associations of SNAP25 and SNAP-25/A 342 with the incidence of $\mathrm{AD}$ were assessed by calculating hazard ratios (HRs) with 95\% confidence intervals (CIs) using Cox proportional hazard regression analysis with adjustment for age and sex. SNAP25 and SNAP-25/A 342 were categorized into two groups by the median of each biomarker when conducting Cox proportional hazard regression analysis.

For MMSE, ADAS-cog, hippocampal and ventricular volumes, and $\mathrm{WMH}$, intercepts (baseline values) and slopes (rates of change) were derived using linear mixed effects models. The intercept and slopes were then used as outcomes in linear regression models with SNAP-25 and SNAP-25/A 342 as predictors (adjusted for age and gender; and for education for MMSE and ADAS-cog; and for intracranial volume for hippocampal and ventricular volumes) within diagnostic groups. All statistics were performed using R (v. 3.4.2) and SPSS version 20. Statistical significance was defined as $p<0.05$ for all analyses.

\section{Results}

\section{Demographic results}

The demographics and biomarker characteristics of the study subjects are presented in Table 1 . There were no 
Table 1 Demographics of subjects at baseline

\begin{tabular}{|c|c|c|c|c|}
\hline Characteristics & $\mathrm{CN}(n=52)$ & $\operatorname{sMCl}(n=22)$ & $\mathrm{pMCl}(n=47)$ & $\mathrm{AD}(n=18)$ \\
\hline Age (years) & $76.2(5.1)$ & $76.0(5.1)$ & $73.1(6.6)$ & $74.3(7.0)$ \\
\hline Gender, female (\%) & $22(42.3 \%)$ & $7(31.8 \%)^{d}$ & $14(29.8 \%)^{d}$ & $11(61.1 \%)$ \\
\hline Education (years) & $15.7(3.3)$ & $16.8(2.4)$ & $15.9(2.7)$ & $15.2(2.9)$ \\
\hline APOE $\varepsilon 4, n(\%)$ & $10(19.2 \%)^{c, d}$ & $7(31.8 \%)^{c, d}$ & $28(59.6 \%)^{a, b}$ & $13(72.2 \%)^{a, b}$ \\
\hline MMSE & $29.3(1.0)^{b, c, d}$ & $27.2(1.4)^{\mathrm{a}, \mathrm{d}}$ & $26.6(1.6)^{\mathrm{a}, \mathrm{d}}$ & $24.2(2.1)^{\mathrm{a}, \mathrm{b}, \mathrm{c}}$ \\
\hline ADAS-cog & $5.9(2.8)^{b, c, d}$ & $9.2(3.8)^{a, c, d}$ & $12.5(4.3)^{\mathrm{a}, \mathrm{b}, \mathrm{d}}$ & $18.2(6.1)^{\mathrm{a}, \mathrm{b}, \mathrm{c}}$ \\
\hline Aß42 (pg/ml) & $211.4(56.2)^{b, c, d}$ & $181.0(54.7)^{a, c, d}$ & $147.5(47.8)^{\mathrm{a}, \mathrm{b}}$ & $136.1(27.3)^{\mathrm{a}, \mathrm{b}}$ \\
\hline t-tau (pg/ml) & $72.7(28.5)^{c, d}$ & $84.7(54.0)^{d}$ & $107.8(49.3)^{\mathrm{a}, \mathrm{d}}$ & $153.2(78.7)^{a, b, c}$ \\
\hline t-tau/Aß42 & $0.399(0.283)^{c, d}$ & $0.553(0.492)^{c, d}$ & $0.820(0.501)^{a, b, d}$ & $1.138(0.569)^{\mathrm{a}, \mathrm{b}, \mathrm{c}}$ \\
\hline p-tau (pg/ml) & $24.6(10.4)^{c, d}$ & $28.1(15.7)^{c, d}$ & $39.5(16.5)^{a, b}$ & $45.8(16.4)^{a, b}$ \\
\hline p-tau/Aß42 & $0.135(0.095)^{c, d}$ & $0.184(0.147)^{c, d}$ & $0.303(0.162)^{a, b}$ & $0.350(0.145)^{a, b}$ \\
\hline Follow-up (years) & $7.5(2.6)^{b, c, d}$ & $4.8(2.5)^{\mathrm{a}, \mathrm{d}}$ & $5.7(2.6)^{\mathrm{a}, \mathrm{d}}$ & $2.6(0.7)^{a, b, c}$ \\
\hline
\end{tabular}

Values are shown as mean \pm standard deviation unless otherwise indicated

$p$ values indicate the values assessed with analyses of variance for each variable except gender and APOE $\varepsilon 4$, where a contingency chi-square was performed. Post-hoc analysis provided significant differences between groups: ${ }^{a}$ versus $\mathrm{CN} ;{ }^{b}$ versus sMCl; ${ }^{c}$ versus $\mathrm{pMCl}^{\mathrm{d}}{ }^{\mathrm{d}}$ versus $\mathrm{AD}$.

$A \beta$ amyloid- $\beta, A D$ Alzheimer's disease, ADAS-cog Alzheimer's Disease Assessment Scale cognitive subscale, APOE apolipoprotein E, CN cognitively normal, MMSE Mini-Mental State Examination, $p M C l$ progressive mild cognitive impairment, $p$-tau phosphorylated tau, $s M C l$ stable mild cognitive impairment, $t$-tau total tau

differences in age or educational level among the groups. Some group cohorts differed significantly for gender, percentage of individuals with the APOE $\varepsilon 4$ genotype, mean MMSE, mean ADAS-cog, biomarker levels, and follow-up time (Table 1).
CSF SNAP-25 levels and SNAP-25/Aß42 ratio in different diagnostic groups

CSF SNAP-25 levels were significantly higher in patients with pMCI $(p<0.01)$ and $\mathrm{AD}(p<0.0001)$ compared with CN. Higher SNAP-25 levels were also found in

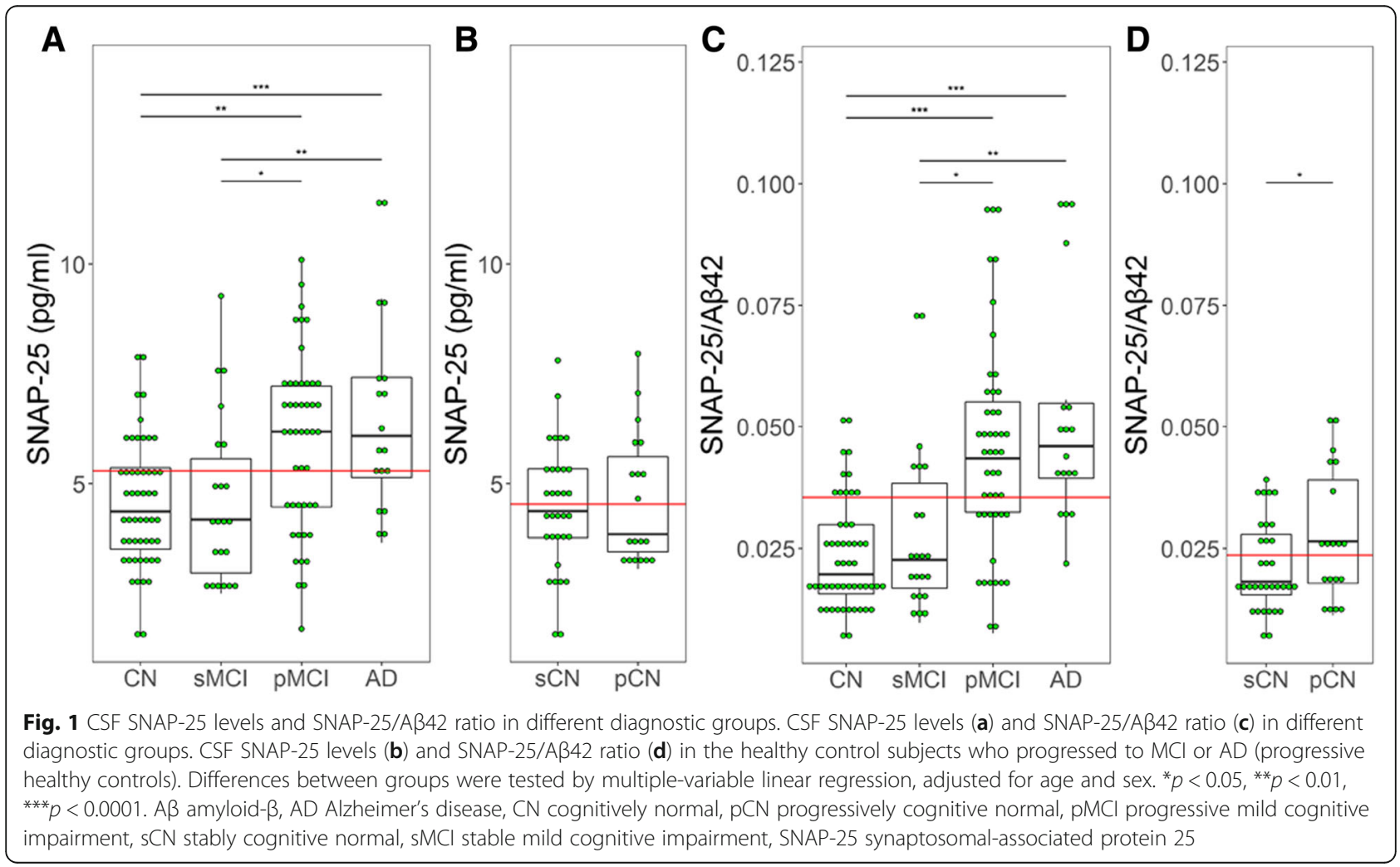


both pMCI $(p=0.037)$ and $\mathrm{AD}(p<0.01)$ compared with sMCI. However, there were no differences between $\mathrm{CN}$ and sMCI, and similarly between pMCI and AD (Fig. 1a). SNAP-25/A 342 ratio also showed the same trend in the different diagnostic groups (Fig. 1c). Among the CN, 19 individuals progressed to $\mathrm{MCI}$ or $\mathrm{AD}$ during follow-up (progressively cognitive normal $(\mathrm{pCN})$ ). These participants had significantly higher SNAP-25/A 442 ratio $(p=0.039)$ compared with stably cognitive normal $(\mathrm{sCN})$ individuals who did not progress to MCI or AD (Fig. 1d). However, The $\mathrm{A} \beta 42$ levels for $\mathrm{pCN}$ are less than that of $\mathrm{sCN}(p=$ 0.042, data not shown). SNAP-25 levels were similar between $\mathrm{sCN}$ and $\mathrm{pCN}$ (Fig. 1b).

\section{Associations between CSF SNAP-25 and A $\beta$ pathology}

To evaluate the correlations between SNAP-25 and A $\beta$ pathology, participants were dichotomized into CSF $\mathrm{A} \beta$-positive $\left(\mathrm{A} \beta^{+}\right)$and $A \beta$-negative $\left(A \beta^{-}\right)$using the previously established cutoff for CSF A $\beta 42$ of $192 \mathrm{pg} / \mathrm{ml}$ [20]: $\mathrm{CN} \mathrm{A} \beta^{-}(n=35), \mathrm{CN} \mathrm{A} \beta^{+}(n=17), s M C I A \beta^{-}(n=8)$, $\operatorname{sMCI~A} \beta^{+}(n=14), \mathrm{pMCI} A \beta^{-}(n=6), \mathrm{pMCI} \mathrm{A} \beta^{+}(n=41)$,

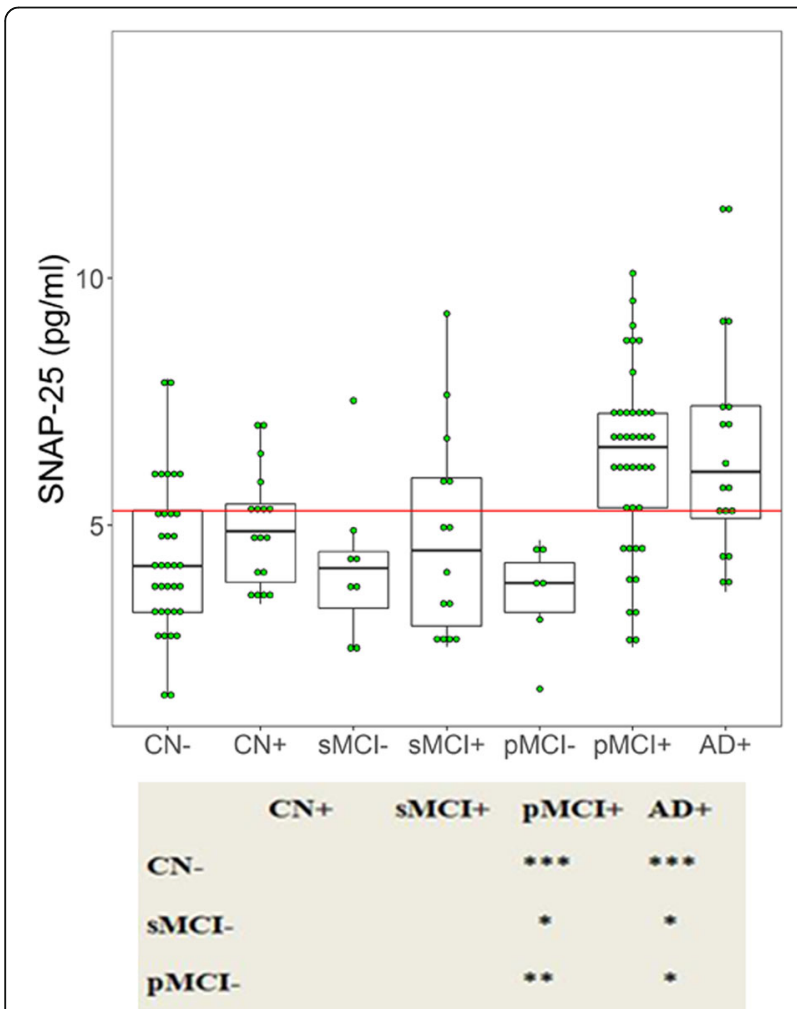

Fig. 2 CSF SNAP-25 by diagnosis and amyloid pathology. The subjects included in the study were classified as $A \beta^{+}$or $A \beta^{-}$. CSF SNAP-25 in different combinations of clinical diagnosis and $A \beta$ pathology. Differences between groups were tested by multiple-variable linear regression, adjusted for age and sex. ${ }^{*} p<0.05,{ }^{* *} p<0.01$, ${ }^{* * *} p<0.0001$. AD Alzheimer's disease, $C \mathrm{~N}$ cognitively normal, $\mathrm{pMCl}$ progressive mild cognitive impairment, $\mathrm{sMCl}$ stable mild cognitive impairment, SNAP-25 synaptosomal-associated protein 25 and $\mathrm{AD} \mathrm{A} \beta^{+}(n=18)$. There were no participants with $\mathrm{AD}$ $\mathrm{A} \beta^{-}$in this study. $\mathrm{pMCI} A \beta^{+}$and $\mathrm{AD} \mathrm{A} \beta^{+}$patients had increased SNAP-25 levels compared with $\mathrm{CN} \mathrm{A} \beta^{-}(p<$ 0.0001 for both groups) (Fig. 2). Furthermore, pMCI $A \beta^{+}$ and $\mathrm{AD} \mathrm{A} \beta^{+}$cases had increased SNAP-25 levels compared with sMCI A $\beta^{-}$( $p=0.025$ and $p=0.01$, respectively) and $\mathrm{pMCI} \mathrm{A} \beta^{-}(p=0.004$ and $p=0.036$, respectively) subjects. sMCI $A \beta^{-}$and pMCI $A \beta^{-}$subjects had SNAP-25 levels in the same range as $\mathrm{CNA} \beta^{-}$patients (Fig. 2).

There were no significant associations between SNAP-25 and A 342 in CN, sMCI, or AD subjects $(r=$ $-0.092, p=0.518 ; r=-0.324, p=0.142 ; r=0.121, p=$ 0.633 ; respectively). SNAP- 25 and A $\beta 42$ were negatively correlated in pMCI patients $(r=-0.389, p=0.007)$ (Fig. 3). By nature of being a ratio with A $\beta 42$, SNAP-25/ $\mathrm{A} \beta 42$ will show a difference between $\mathrm{A} \beta 42^{+}$and $\mathrm{A} \beta 42^{-}$ subjects. Therefore, we did not analyze the correlations between SNAP-25/A $\beta 42$ ratio and $A \beta$ pathology.

\section{Associations between CSF SNAP-25 and SNAP-25/A 342 and tau biomarkers}

$\mathrm{t}$-tau and p-tau were strongly correlated with SNAP-25 in $\mathrm{CN}(r=0.483, p<0.001$ for $\mathrm{t}$-tau; $r=0.522, p<0.001$ for p-tau), sMCI $(r=0.876, p<0.001$ for t-tau; $r=0.858$, $p<0.001$ for $\mathrm{p}$-tau), pMCI $(r=0.642, p<0.001$ for $\mathrm{t}$-tau; $r=0.528, p<0.001$ for $\mathrm{p}$-tau), and $\mathrm{AD}$ subjects $(r=$ 0.791, $p<0.001$ for $\mathrm{t}$-tau; $r=0.644, p=0.004$ for $\mathrm{p}$-tau) (Fig. $4 \mathrm{a}, \mathrm{c})$. t-tau and $\mathrm{p}$-tau were also strongly correlated with SNAP-25/A 342 in all groups (Fig. 4b, d).

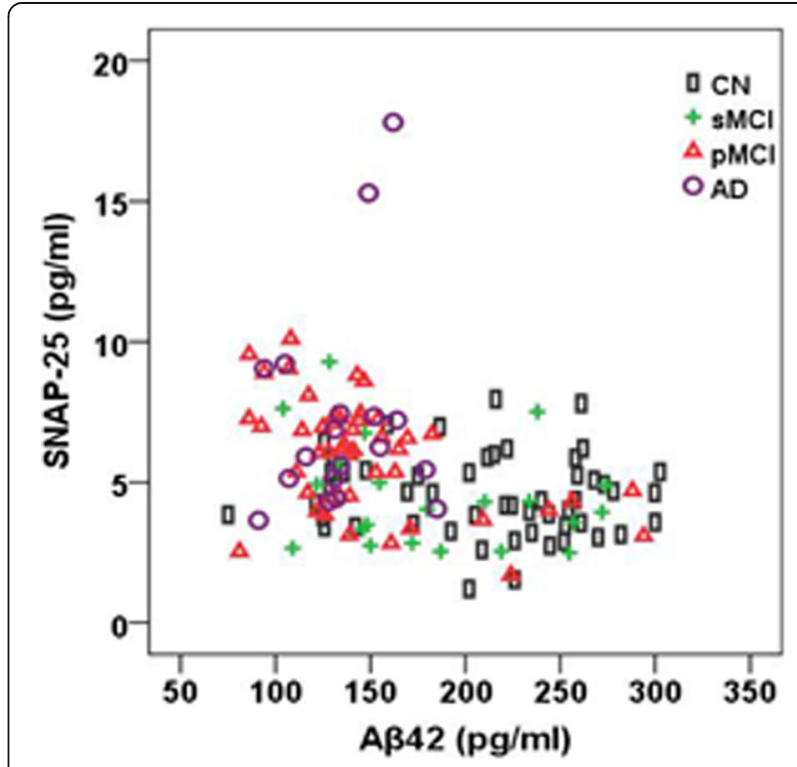

Fig. 3 CSF SNAP-25 levels in relation to A 442 . Correlations between CSF SNAP-25 levels and $A \beta 42$ in different diagnostic groups. $A \beta$ amyloid- $\beta, A D$ Alzheimer's disease, $C N$ cognitively normal, $\mathrm{pMCl}$ progressive mild cognitive impairment, $s \mathrm{MCl}$ stable mild cognitive impairment, SNAP-25 synaptosomal-associated protein 25 

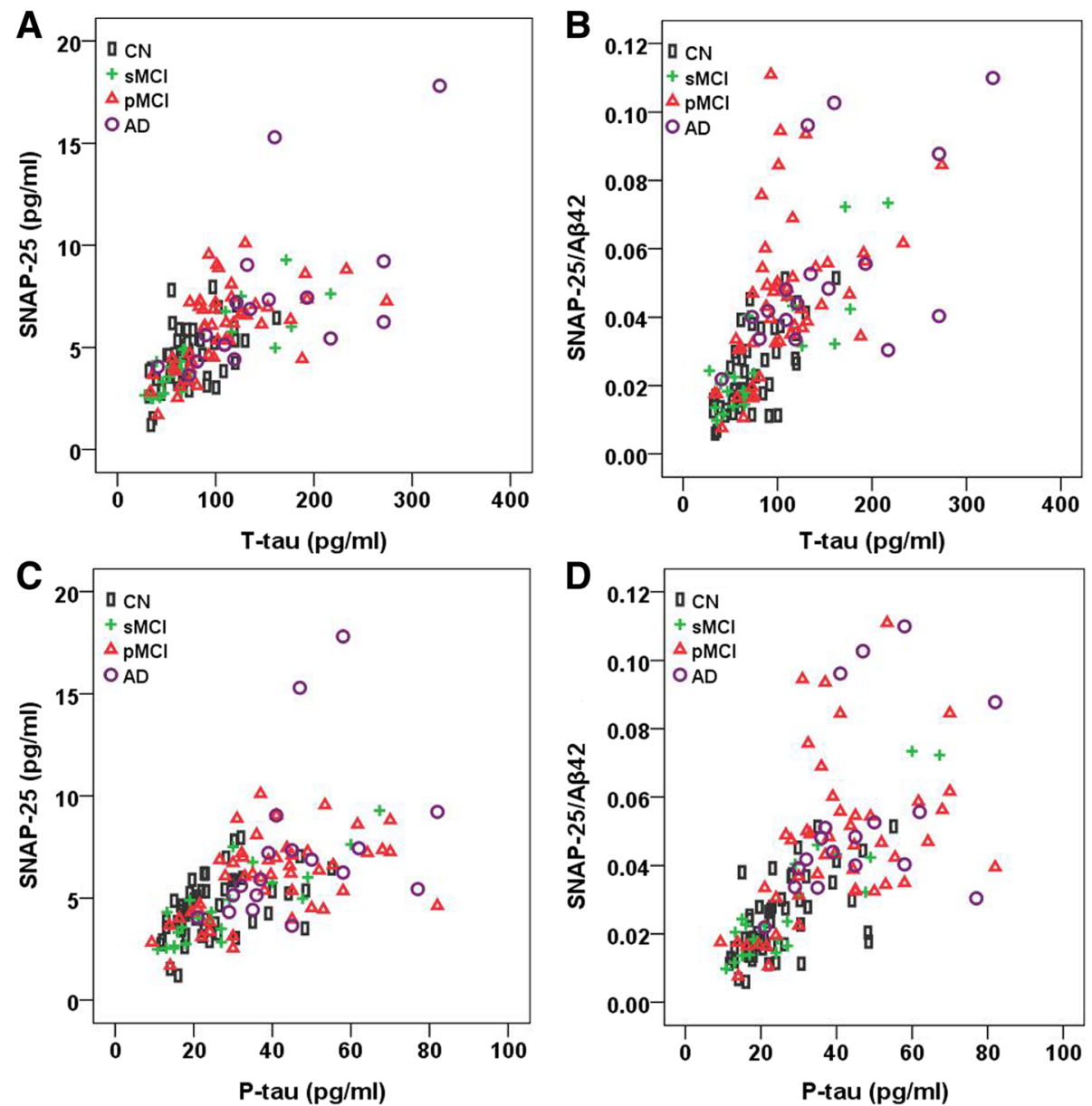

Fig. 4 CSF SNAP-25 levels and SNAP-25/Aß42 ratio in relation to tau biomarkers. Correlations between CSF SNAP-25 levels (a) and SNAP-25/Aß42 ratio (b) and t-tau in different diagnostic groups. Correlations between CSF SNAP-25 levels (c) and SNAP-25/Aß42 ratio (d) and p-tau in different diagnostic groups. A $\beta$ amyloid- $\beta$, AD Alzheimer's disease, $C N$ cognitively normal, $P$-tau phosphorylated tau, pMCl progressive mild cognitive impairment, sMCl stable mild cognitive impairment, SNAP-25 synaptosomal-associated protein 25, T-tau total tau

Diagnostic accuracy of CSF SNAP-25, SNAP-25/Aß42, and core CSF biomarkers

ROC analyses were performed to test CSF biomarkers and ratios in relation to clinical diagnoses for $\mathrm{pMCI}$ and AD. All CSF biomarkers and ratios had significant diagnostic accuracy for pMCI (Table 2 and Fig. 5a) and AD (Table 2 and Fig. 5b) compared with CN. Compared with A $\beta 42$, t-tau, and p-tau, SNAP-25 had almost the same range of diagnostic accuracy for pMCI (SNAP-25 vs $\mathrm{A} \beta 42, p=0.21$; SNAP-25 vs t-tau, $p=0.60$; SNAP-25 vs p-tau, $p=0.18$ ) (Table 2 and Fig. 5a) and $\mathrm{AD}$ (SNAP-25 vs A $342, p=0.41$; SNAP-25 vs t-tau, $p=0.11$; SNAP-25 vs p-tau, $p=0.07$ ) (Table 2 and Fig. 5b). Similarly, the SNAP-25/A 342 ratio appears to offer diagnostic accuracy at least as well as t-tau/A $\beta 42$ and p-tau/ $\mathrm{A} \beta 42$ for $\mathrm{pMCI}$ (SNAP-25/A $\beta 42$ vs $\mathrm{t}$-tau/A $\beta 42, p=0.88$; SNAP-25/A $\beta 42$ vs $p$-tau/A $\beta 42, p=0.93$ ) (Table 2 and Fig. 5a) and AD (SNAP-25/A $\beta 42$ vs t-tau/A $\beta 42, p=0.79$; SNAP-25/A $\beta 42$ vs p-tau/A $\beta 42, p=0.81$ ) (Table 2 and Fig. 5b). In addition, SNAP-25/A $\beta 42$ provided higher

Table 2 AUC of CSF biomarkers

\begin{tabular}{|c|c|c|c|c|c|c|c|}
\hline & SNAP-25 & Aß42 & t-tau & p-tau & SNAP-25/Aß42 & t-tau/Aß42 & $\mathrm{p}-\operatorname{tau} / \mathrm{A} \beta 42$ \\
\hline $\mathrm{pMCl}$ & $\begin{array}{l}0.72(0.61-0.82) \\
(p<0.001)\end{array}$ & $\begin{array}{l}0.80(0.70-0.89) \\
(p<0.001)\end{array}$ & $\begin{array}{l}0.74(0.65-0.84) \\
(p<0.001)\end{array}$ & $\begin{array}{l}0.78(0.69-0.88) \\
(p<0.001)\end{array}$ & $\begin{array}{l}0.81(0.72-0.90) \\
(p<0.001)\end{array}$ & $\begin{array}{l}0.81(0.72-0.90) \\
(p<0.001)\end{array}$ & $\begin{array}{l}0.81(0.72-0.90) \\
(p<0.001)\end{array}$ \\
\hline$A D$ & $\begin{array}{l}0.79(0.67-0.91) \\
(p=0.002)\end{array}$ & $\begin{array}{l}0.85(0.77-0.94) \\
(p<0.001)\end{array}$ & $\begin{array}{l}0.87(0.75-0.98) \\
(p<0.001)\end{array}$ & $\begin{array}{l}0.88(0.80-0.96) \\
(p<0.001)\end{array}$ & $\begin{array}{l}0.91(0.84-0.98) \\
(p<0.001)\end{array}$ & $\begin{array}{l}0.91(0.82-1.00) \\
(p<0.001)\end{array}$ & $\begin{array}{l}0.91(0.84-0.98) \\
(p<0.001)\end{array}$ \\
\hline
\end{tabular}

$A \beta$ amyloid- $\beta, A D$ Alzheimer's disease, $A U C$ area under the receiver operator characteristics curve, CSF cerebrospinal fluid, $p M C l$ progressive mild cognitive impairment, $p$-tau phosphorylated tau, SNAP-25 synaptosomal-associated protein $25, t$-tau total tau 

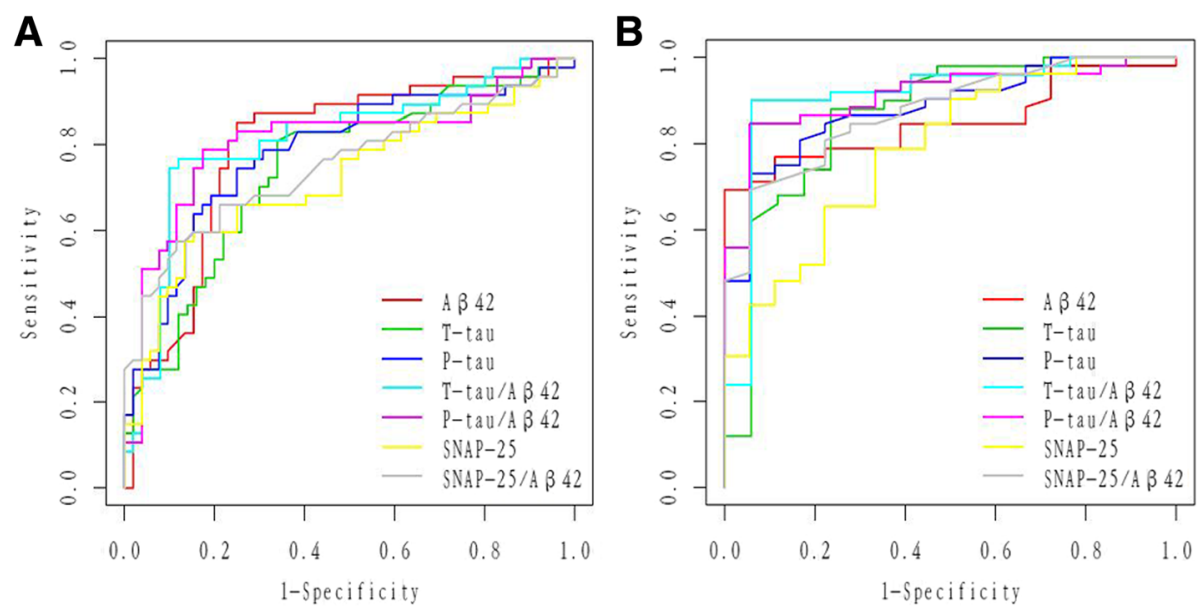

Fig. 5 ROC analyses. ROC analyses were performed to test the CSF biomarkers and ratios in relation to clinical diagnoses for $\mathrm{pMCl}$ (a) and $A D(\mathbf{b})$. A $\beta$ amyloid- $\beta$, P-tau phosphorylated tau, SNAP-25 synaptosomal-associated protein 25, T-tau total tau

diagnostic accuracy than SNAP-25 alone for $\mathrm{pMCI}(p=$ 0.013) (Table 2 and Fig. 5a) and AD ( $p=0.015)$ (Table 2 and Fig. 5b). However, t-tau/A 442 and $p$-tau/A 442 ratios provided higher diagnostic accuracy than SNAP-25 alone for $\mathrm{AD}(p=0.044$ and $p=0.038$, respectively) (Table 2 and Fig. 5b) but not pMCI ( $p=0.06$ for both) (Table 2 and Fig. 5a).

\section{CSF SNAP-25 and SNAP-25/A 342 predict conversion from $\mathrm{MCl}$ to $\mathrm{AD}$}

We investigated whether CSF SNAP-25 and SNAP25/A 342 predicted conversion from MCI to AD. Cox proportional hazard models were performed for SNAP-25 and SNAP-25/A 342 as a continuous variable after adjusting for age and gender. CSF SNAP-25 and SNAP-25/A 342 significantly predicted conversion from $\mathrm{MCI}$ to $\mathrm{AD}$. HRs were then calculated for SNAP-25 and SNAP-25/A $\beta 42$ as a dichotomous variable using the median values of SNAP-25 and SNAP-25/A 342 as a cutoff (adjusting for age and gender). Individuals with high SNAP-25 (HR 2.47, $p=$ 0.011 ), corresponding to individuals whose SNAP-25 values were $\geq 5.4 \mathrm{pg} / \mathrm{ml}$, progressed much more rapidly to $\mathrm{AD}$ than individuals with lower values $(<$ $5.4 \mathrm{pg} / \mathrm{ml}$, corresponding to the lower median values of SNAP-25) (Fig. 6a). Individuals with high SNAP-25/A 342 (HR 2.41, $p=0.013$ ), corresponding to individuals whose SNAP-25/A $\beta 42$ values were $\geq 0.037$,
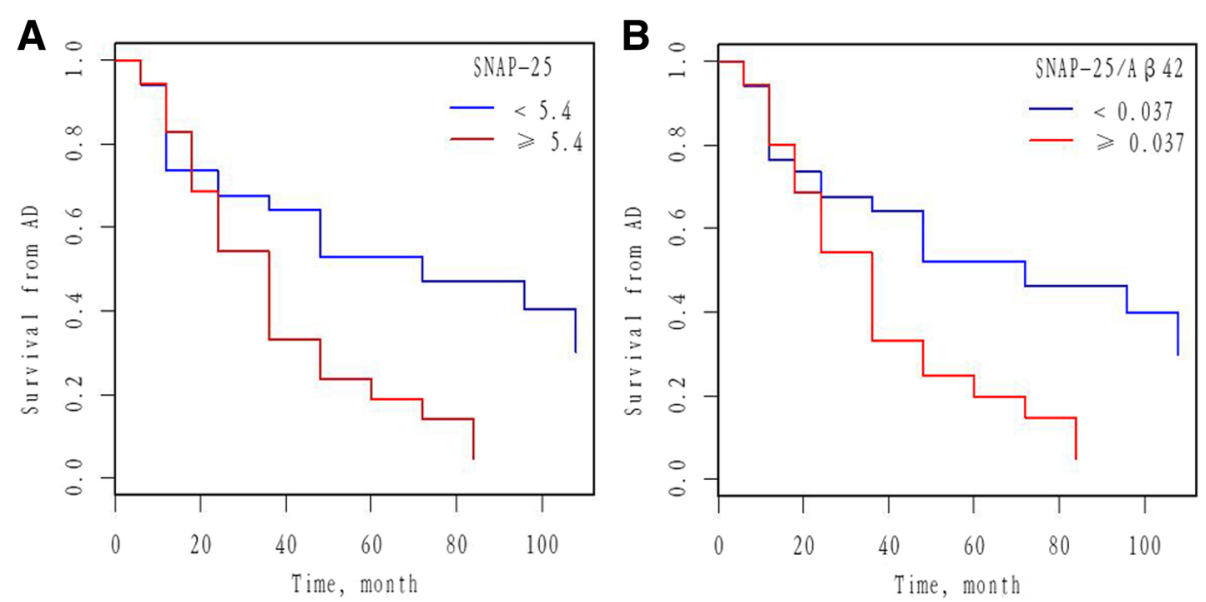

Fig. 6 Baseline CSF measures of SNAP-25 and SNAP-25/Aß42 as predictors of conversion from MCI to AD. Survival from AD as a function of CSF SNAP-25 (a) and SNAP-25/Aß42 (b) measures (dichotomized at the median values) are shown. Analyses were adjusted for age and gender. Cutoff values were $5.4 \mathrm{pg} / \mathrm{ml}$ and 0.037 for SNAP-25 and SNAP-25/Aß42, respectively. A $\beta$ amyloid- $\beta$, AD Alzheimer's disease, SNAP-25 synaptosomal-associated protein 25 

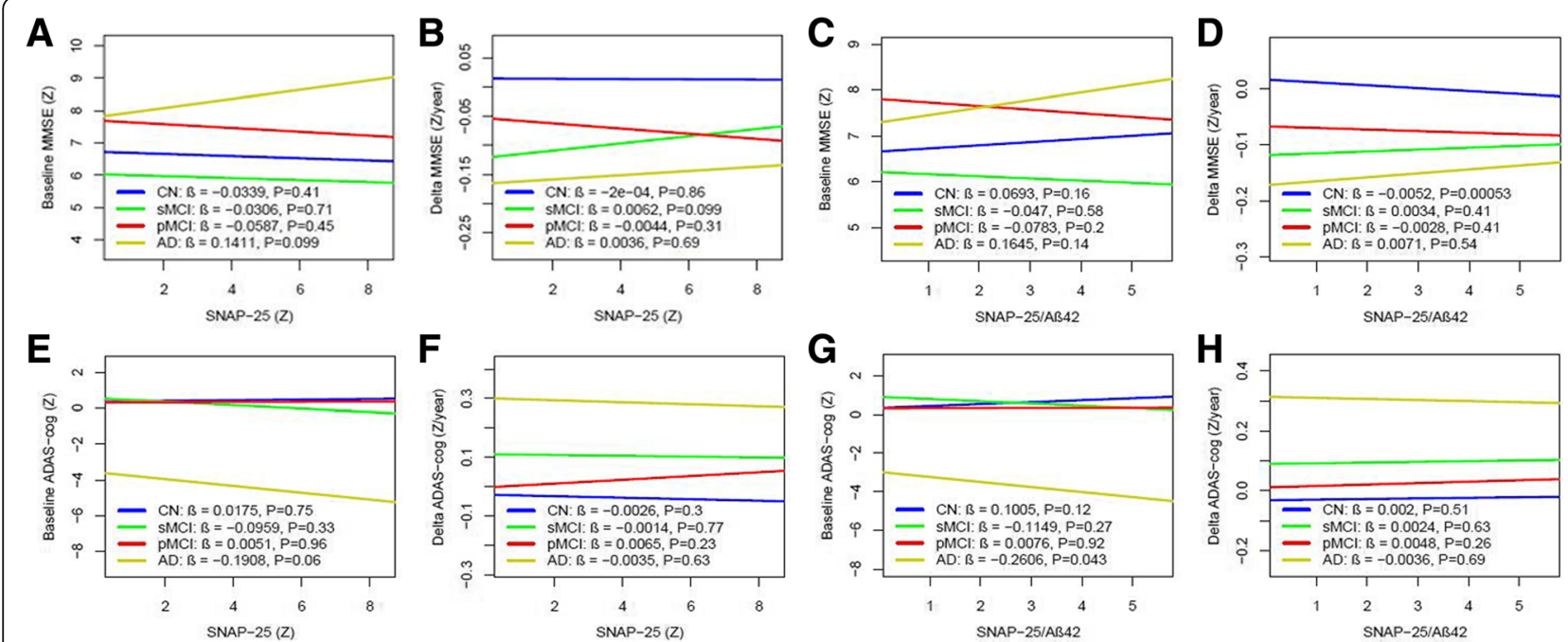

Fig. 7 CSF SNAP-25 and SNAP-25/Aß42 in relation to cognition and future cognitive change. MMSE and ADAS-cog at baseline (a, e) and over time $(\mathbf{b}, \mathbf{f})$ as a function of baseline CSF SNAP-25 in different diagnostic groups. MMSE and ADAS-cog at baseline $(\mathbf{c}, \mathbf{g})$ and over time $(\mathbf{d}, \mathbf{h})$ as a function of baseline SNAP-25/A 42 in different diagnostic groups. Biomarker levels and ratios are standardized to $z$ scores. A $\beta$ amyloid- $\beta$, AD Alzheimer's disease, ADAS-cog Alzheimer's Disease Assessment Scale cognitive subscale, CN cognitively normal, MMSE Mini-Mental State Examination, $\mathrm{pMCl}$ progressive mild cognitive impairment, sMCI stable mild cognitive impairment, SNAP-25 synaptosomal-associated protein 25

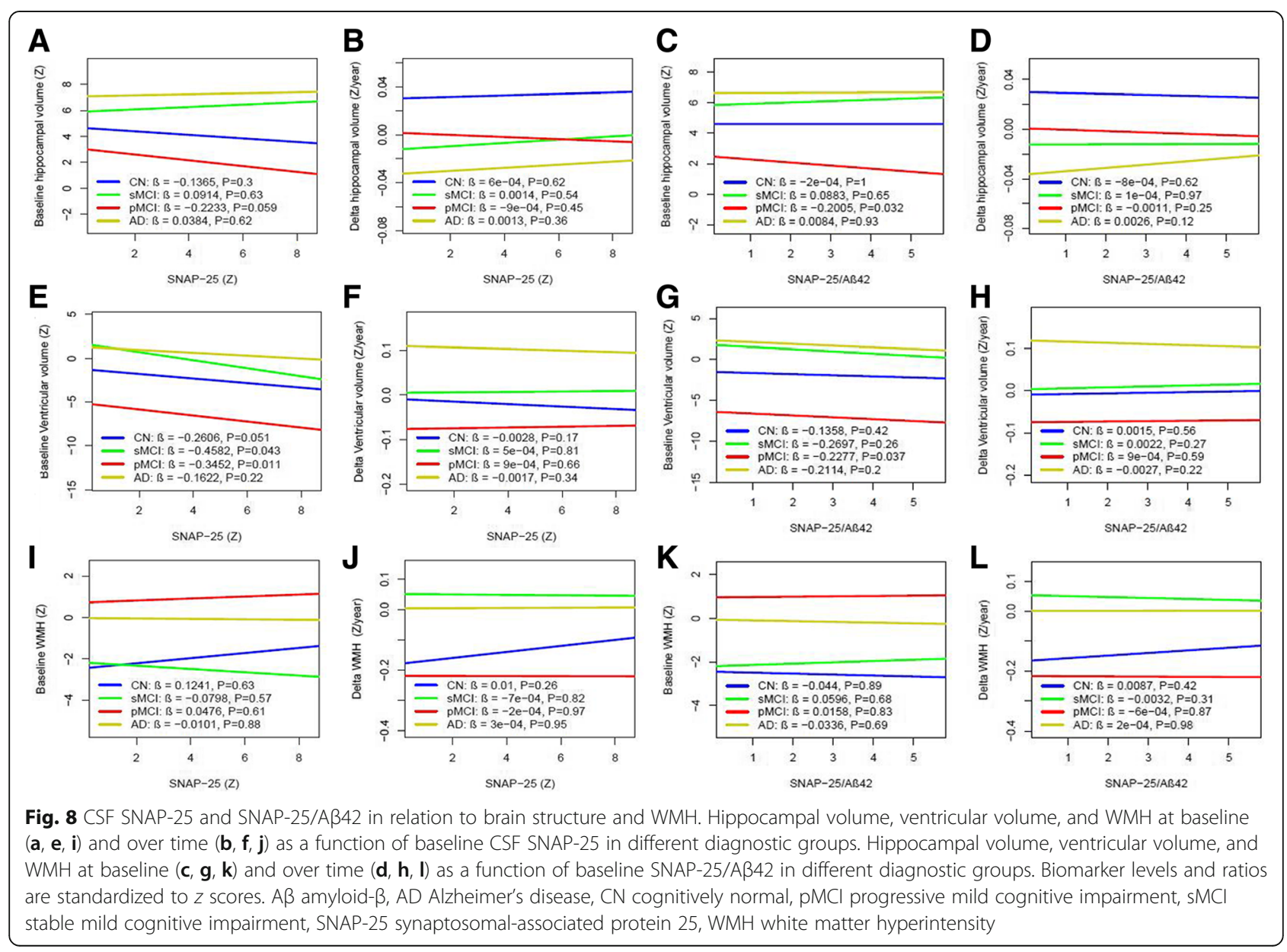


progressed much more rapidly to $\mathrm{AD}$ than individuals with lower values $(<0.037$, corresponding to the lower median values of SNAP-25/Aß42) (Fig. 6b).

\section{CSF SNAP-25 and SNAP-25/Aß42 in relation to cognition} CSF SNAP-25 did not correlate with MMSE or ADAS-cog scores at baseline or rates of change of MMSE and ADAS-cog during follow-up (Fig. 7a, b, e, f). In contrast, SNAP-25/A $\beta 42$ ratio correlated with ADAS-cog scores in AD at baseline $(\beta=-0.26, p=$ 0.043) (Fig. $7 \mathrm{~g}$ ) and a decreased changing rate of MMSE for $\mathrm{CN}$ during the clinical follow-up period $(\beta=-0.01, p<0.001)$ (Fig. $7 \mathrm{~d}$ ).

\section{CSF SNAP-25 and SNAP-25/A 342 in relation to brain structure and WMH}

Finally, we examined whether SNAP-25 and SNAP-25/ A 342 correlated with hippocampal volumes, ventricular volumes, and WMH as measured with MRI (Fig. 8). SNAP-25 was associated with baseline smaller ventricular volumes in sMCI $(\beta=-0.46, p=0.043)$ and $\mathrm{pMCI}(\beta$ $=-0.35, p=0.011$ ) groups (Fig. 8e), but was not associated with baseline hippocampal volumes or WMH in any group (Fig. 8a, i). SNAP-25 was not associated with rates of change of brain structure or WMH during the follow-up period in any group (Fig. 8b, i, j). SNAP-25/ A $\beta 42$ ratio was associated with baseline hippocampal ( $\beta$ $=-0.20, p=0.032$ ) (Fig. 8c) and smaller ventricular volumes $(\beta=-0.23, p=0.037$ ) (Fig. 8g) in the pMCI group.

\section{Discussion}

The present study investigated the relationships between SNAP-25, a biomarker of synaptic loss, and other key biomarkers across the $\mathrm{AD}$ spectrum. The results demonstrate that SNAP-25 markers increase with disease severity and are able to distinguish between diagnostic groups. Finally, markers of synapse loss were predictive of clinical progression to dementia and neurodegeneration.

For many years, the classic definition of neurodegenerative diseases such as AD was limited to the discovery of selective neuronal loss and astrogliosis. This concept, however, has now been extended to include neuroinflammation and synaptic loss [9]. Synaptic loss precedes neuronal loss and correlates with early deficits in memory formation [22]. SNAP-25 is a characteristic component of synapses and is highly expressed in the central nervous system. The absence of SNAP-25 may not stop neurotransmission, but it is associated with several physiological functions including synaptic vesicle release and recycling, neurite extension, neuron repair, and synaptogenesis [23, 24]. SNAP-25 is involved in the regulation of long-term potentiation and formation of long-term memory in the hippocampus CA3 region, which is consistent with its role in learning and memory functions in the hippocampal CA1 area [25]. Since previous studies have shown that SNAP-25 was detectable in CSF [26], this presynaptic protein can be used as a biomarker to monitor the molecular pathogenesis of $\mathrm{AD}$ that was previously difficult to assess in living patients.

CSF SNAP-25 was significantly increased in pMCI and $\mathrm{AD}$ compared with the cognitively normal. In the present study, we also show that pMCI patients had increased CSF SNAP-25 levels compared with sMCI individuals. SNAP-25/AB42 ratio had the same trend in every diagnostic group. Interestingly, 19 cognitively normal subjects who progressed to $\mathrm{MCI}$ or $\mathrm{AD}$ during follow-up had increased SNAP-25/A 342 ratio (but not SNAP-25) compared with the other cognitively normal subjects. This finding suggests that CSF SNAP-25, especially the SNAP-25/A $\beta 42$ ratio, is an early pathophysiological indicator of AD-related synaptic loss.

To investigate whether CSF SNAP-25 levels correlated with underlying $A \beta$ pathology, each group included in the study was dichotomized into $A \beta^{+}$and $A \beta^{-}$. As expected, the CSF SNAP-25 levels were significantly increased in the CSF AD A $\beta^{+}$group. Similarly, high CSF SNAP-25 levels were also found in the pMCI A $\beta^{+}$group. In addition, SNAP-25 and A $\beta 42$ were negatively correlated in pMCI patients. Currently, the general view is that $\mathrm{AD}$ progresses from $\mathrm{CN} \mathrm{A} \beta^{-}$to $\mathrm{CN} \mathrm{A} \beta^{+}, \mathrm{MCI} A \beta^{+}$, and finally $A D A \beta^{+}$, while non-A $\beta$-dependent cognitive decline may progress from $C N A \beta^{-}$to $M C I A \beta^{-}$and $A D$ $A \beta^{-}$[27]. In addition, $t$-tau and $p$-tau were strongly correlated with SNAP-25 and SNAP-25/A 342 ratio in every diagnostic group, suggesting that SNAP-25 [28] or SNAP-25/A 42 ratio may be a surrogate biomarker for future clinical treatment studies with tau-modifying drugs. Interestingly, we observed the strongest correlation between SNAP-25 and tau in sMCI patients. We do not know the specific reason for this, but we speculate that tau protein pathology plays a more important role in the pathogenesis of sMCI.

In the present study, our results show that CSF SNAP-25, especially SNAP-25/A 42 , offers diagnostic sensitivity for $\mathrm{AD}$ that is comparable with that of CSF $\mathrm{A} \beta 42$, $\mathrm{t}$-tau, $\mathrm{p}$-tau, $\mathrm{t}$-tau/A $\beta 42$, and $\mathrm{p}$-tau/A $\beta 42$. CSF SNAP-25 and SNAP-25/A 442 also have high diagnostic sensitivity for pMCI. In addition, SNAP-25/A 342 appears to offer diagnostic sensitivity at least as well as the current "gold standard" prognostic biomarkers for $\mathrm{AD}$, such as $\mathrm{t}$-tau/A $\beta 42$ and $\mathrm{p}$-tau/A 342 . These findings, therefore, suggest CSF SNAP-25 and SNAP-25/A 342 ratio as diagnostic biomarkers for the earliest symptomatic stage of $\mathrm{AD}$.

There is accumulating evidence that synaptic loss is a surrogate for disease progression in AD [7]. To the best of our knowledge, no studies have evaluated the predictive value of SNAP-25 for the conversion from MCI to 
AD. Here, we demonstrate that CSF SNAP-25 and SNAP-25/A 342 offer predictive value for future disease progression in $\mathrm{MCI}$ subjects. Our findings suggest that CSF SNAP-25 and SNAP-25/A 342 may complement the prognostic utility of CSF $A \beta 42$, $t$-tau, and p-tau in predicting the evolution of cognitive impairment.

Evidence suggests that progressive neuronal and synaptic loss are the surrogate markers for cognitive deterioration in AD [7]. In the present study, although CSF SNAP-25 does not correlate with cognitive decline at baseline and follow-up, our results suggest that the SNAP-25/A 342 ratio may offer predictive value for future cognitive impairment in cognitively normal subjects. In our study, 19 cognitively normal subjects who progressed to MCI or AD during follow-up had increased SNAP-25/A 342 ratio compared with stably cognitive normal subjects, which implies that the combination of SNAP-25 and A 342 (SNAP-25/Aß42) might be useful to follow progression of cognitive decline. However, SNAP-25 levels were similar between stably cognitive normal subjects and progressively cognitive normal subjects, and A 342 levels in progressively cognitive normal subjects were less than those in stably cognitive normal subjects. Thus, these results do not suggest that SNAP-25 has added predictive value on top of A $\beta 42$ alone for cognitively normal subjects. We also found associations between high SNAP-25/A 342 ratio and an increased rate of hippocampal atrophy. Given that synaptic dysfunction and loss are probably the major causes of the loss of neuropil underlying hippocampal atrophy [12], we believe that SNAP-25/A 342 may be an independent novel biomarker for synaptic pathology in $\mathrm{AD}$, and the clinical manifestations of cognitive impairment and later dementia appear after neuronal injury and synaptic loss has reached a threshold in vulnerable brain regions. This study has limitations. First, our cases do not include non-AD neurodegenerative diseases, and our study is not a pathological study; thus it lacks pathological evidence. Second, the ADNI database was volunteered by highly educated individuals for research focused on AD research. This may give rise to bias in choice because the study population is a self-selected individual who may have concerns about their cognition. Third, the convenience samples used in this study may limit the universality of our findings. Finally, the sample size of this study is relatively small. Therefore, it is necessary to replicate our results in larger population-based cohorts and to conduct research on the related mechanisms.

\section{Conclusions}

In summary, CSF SNAP-25, especially the SNAP-25/ A $\beta 42$ ratio, was already increased in the predementia stages of $\mathrm{AD}$, and higher concentrations correlate with a higher rate of cognitive decline and hippocampal atrophy at some stages of AD. These findings may highlight the potential use of CSF SNAP-25 and SNAP-25/A 342 in trial designs, in response to therapies in clinical trials of disease-modifying therapies, in treatment decisions, and outcome assessments, and may complement diagnostic and prognostic information provided by CSF A $\beta 42, t$-tau, and p-tau.

\begin{abstract}
Abbreviations
A $\beta$ : Amyloid- $\beta$; AD: Alzheimer's disease; ADAS-cog: Alzheimer's Disease Assessment Scale cognitive subscale; ADNI: Alzheimer's disease

Neuroimaging Initiative; ANOVA: Analysis of covariance; AUC: Area under the curve; CDR: Clinical Dementia Rating; Cl: Confidence interval; CN: Cognitively normal; CSF: Cerebrospinal fluid; HR: Hazard ratio; MCl: Mild cognitive impairment; MMSE: Mini-Mental State Examination; MRI: Magnetic resonance imaging; pCN: Progressively cognitive normal; PET: Positron emission tomography; pMCl: Progressive mild cognitive impairment; $\mathrm{p}$ tau: Phosphorylated tau; ROC: Receiver operating curve; $\mathrm{SCN}$ : Stably cognitive normal; sMCI: Stable mild cognitive impairment; SNAP-25: Synaptosomalassociated protein 25; t-tau: Total tau; WMH: White matter hyperintensity
\end{abstract}

\section{Acknowledgments}

We are grateful to the First Affiliated Hospital of Chongqing Medical University. We thank the Department of Neurology, the First Affiliated Hospital of Chongqing Medical University.

Data collection and sharing for this project was funded by the Alzheimer's Disease Neuroimaging Initiative (ADNI) (National Institutes of Health Grant U01 AG024904) and DOD ADNI (Department of Defense award number W81XWH-12-2-0012). ADNI is funded by the National Institute on Aging, the National Institute of Biomedical Imaging and Bioengineering, and through generous contributions from the following: AbbVie, Alzheimer's Association; Alzheimer's Drug Discovery Foundation; Araclon Biotech; BioClinica, Inc.; Biogen; Bristol-Myers Squibb Company; CereSpir, Inc.; Cogstate; Eisai Inc.; Elan Pharmaceuticals, Inc.; Eli Lilly and Company; Eurolmmun; F. Hoffmann-La Roche Ltd and its affiliated company Genentech, Inc.; Fujirebio; GE Healthcare; IXICO Ltd.; Janssen Alzheimer Immunotherapy Research \& Development, LLC.; Johnson \& Johnson Pharmaceutical Research \& Development LLC.; Lumosity; Lundbeck; Merck \& Co., Inc.; MesoScale Diagnostics, LLC.; NeuroRx Research; Neurotrack Technologies; Novartis Pharmaceuticals Corporation; Pfizer Inc.; Piramal Imaging; Servier; Takeda Pharmaceutical Company; and Transition Therapeutics. The Canadian Institutes of Health Research is providing funds to support ADNI clinical sites in Canada. Private sector contributions are facilitated by the Foundation for the National Institutes of Health (www.fnih.org). The grantee organization is the Northern California Institute for Research and Education, and the study is coordinated by the Alzheimer's Therapeutic Research Institute at the University of Southern

California. ADNI data are disseminated by the Laboratory for Neuro Imaging at the University of Southern California.

\section{Funding}

This work was supported by the Weston Brain Institute, Canadian Institutes of Health Research (CIHR) (MOP-11-51-31 to PR-N), the Alzheimer's Association (NIRG-12-92090, NIRP-12-259245 to PR-N), Fonds de Recherche du Québec Santé (FRQS; Chercheur Boursier to PR-N), and Clinical key specialist fund, the Department of Neurology, the First Affiliated Hospital of Chongqing Medical University (scholarship to HZ). SG and PR-N are members of the CIHR-CCNA Canadian Consortium of Neurodegeneration in Aging.

\section{Availability of data and materials}

The data supporting the conclusions of this article are available from the corresponding author upon request.

\section{Authors' contributions}

HZ: study concept, design, analysis and interpretation of data, composition of figures, and manuscript drafting. JT: study design, composition of figures, manuscript drafting, and critical review of manuscript for intellectual content. KPN: study design, and analysis and interpretation of data. MSK and TAP: analysis and interpretation of data. PRN and SG: study concept, design, study 
supervision, and critical review of manuscript for intellectual content. All authors read and approved the final manuscript.

\section{Ethics approval and consent to participate}

The ADNI study was approved by the Institutional Review Boards of all the participating institutions. Informed written consent was obtained from all subjects at each center.

\section{Consent for publication}

All authors approved the final manuscript for submission and gave consent for publication.

\section{Competing interests}

The authors declare that they have no competing interests.

\section{Publisher's Note}

Springer Nature remains neutral with regard to jurisdictional claims in published maps and institutional affiliations.

\section{Author details}

${ }^{1}$ Department of Neurology, the First Affiliated Hospital of Chongqing Medical University, Chongqing 400016, China. ${ }^{2}$ The McGill University Research Centre for Studies in Aging, McGill University, Montreal, Canada. ${ }^{3}$ Department of Neurology, National Neuroscience Institute, Singapore, Singapore.

Received: 20 March 2018 Accepted: 12 July 2018

Published online: 16 August 2018

\section{References}

1. DeKosky ST, Scheff SW, Styren SD. Structural correlates of cognition in dementia: quantification and assessment of synapse change. Neurodegeneration. 1996:5:417-21.

2. Terry RD, Masliah E, Salmon DP, Butters N, DeTeresa R, Hill R, Hansen LA Katzman R. Physical basis of cognitive alterations in Alzheimer's disease: synapse loss is the major correlate of cognitive impairment. Ann Neurol. 1991;30:572-80.

3. DeKosky ST, Scheff SW. Synapse loss in frontal cortex biopsies in Alzheimer's disease: correlation with cognitive severity. Ann Neurol. 1990;27:457-64.

4. Furuya TK, Silva PN, Payao SL, Bertolucci PH, Rasmussen LT, De Labio RW, Braga IL, Chen ES, Turecki G, Mechawar N, Mill J, Smith MA. Analysis of SNAP25 mRNA expression and promoter DNA methylation in brain areas of Alzheimer's disease patients. Neuroscience. 2012;220:41-6.

5. Lista S, Hampel H. Synaptic degeneration and neurogranin in the pathophysiology of Alzheimer's disease. Expert Rev Neurother. 2017:17:47-57.

6. Scheff SW, DeKosky ST, Price DA. Quantitative assessment of cortical synaptic density in Alzheimer's disease. Neurobiol Aging. 1990;11:29-37.

7. Ingelsson M, Fukumoto H, Newell KL, Growdon JH, Hedley-Whyte ET, Frosch MP, Albert MS, Hyman BT, Irizarry MC. Early Abeta accumulation and progressive synaptic loss, gliosis, and tangle formation in AD brain. Neurology. 2004;62:925-31.

8. Scheff SW, Sparks L, Price DA. Quantitative assessment of synaptic density in the entorhinal cortex in Alzheimer's disease. Ann Neurol. 1993;34:356-61.

9. Overk CR, Masliah E. Pathogenesis of synaptic degeneration in Alzheimer's disease and Lewy body disease. Biochem Pharmacol. 2014;88:508-16.

10. Counts SE, Alldred MJ, Che S, Ginsberg SD, Mufson EJ. Synaptic gene dysregulation within hippocampal CA1 pyramidal neurons in mild cognitive impairment. Neuropharmacology. 2014:79:172-9.

11. Scheff SW, Price DA, Schmitt FA, DeKosky ST, Mufson EJ. Synaptic alterations in CA1 in mild Alzheimer disease and mild cognitive impairment. Neurology. 2007;68:1501-8.

12. Portelius $E$, Zetterberg $H$, Skillback T, Tornqvist U, Andreasson U, Trojanowski JQ, Weiner MW, Shaw LM, Mattsson N, Blennow K. Cerebrospinal fluid neurogranin: relation to cognition and neurodegeneration in Alzheimer's disease. Brain. 2015:138:3373-85.

13. Pham E, Crews L, Ubhi K, Hansen L, Adame A, Cartier A, Salmon D, Galasko D, Michael S, Savas JN, Yates JR, Glabe C, Masliah E. Progressive accumulation of amyloid-beta oligomers in Alzheimer's disease and in amyloid precursor protein transgenic mice is accompanied by selective alterations in synaptic scaffold proteins. FEBS J. 2010;277:3051-67.
14. Greber S, Lubec G, Cairns N, Fountoulakis M. Decreased levels of synaptosomal associated protein 25 in the brain of patients with Down syndrome and Alzheimer's disease. Electrophoresis. 1999;20:928-34.

15. Brinkmalm A, Brinkmalm G, Honer WG, Frolich L, Hausner L, Minthon L, Hansson O, Wallin A, Zetterberg H, Blennow K, Ohrfelt A. SNAP-25 is a promising novel cerebrospinal fluid biomarker for synapse degeneration in Alzheimer's disease. Mol Neurodegener. 2014;9:53.

16. Berg L. Clinical dementia rating (CDR). Psychopharmacol Bull. 1988;24:637-9.

17. Folstein MF, Folstein SE, McHugh PR. "Mini-mental state". A practical method for grading the cognitive state of patients for the clinician. J Psychiatr Res. 1975;12:189-98.

18. Aisen PS, Petersen RC, Donohue MC, Gamst A, Raman R, Thomas RG, Walter S, Trojanowski JQ, Shaw LM, Beckett LA, Jack CR Jr, Jagust W, Toga AW, Saykin AJ, Morris JC, Green RC, Weiner MW. Clinical core of the Alzheimer's disease neuroimaging initiative: progress and plans. Alzheimers Dement. 2010;6:239-46.

19. Tierney MC, Fisher RH, Lewis AJ, Zorzitto ML, Snow WG, Reid DW, Nieuwstraten P. The NINCDS-ADRDA work group criteria for the clinical diagnosis of probable Alzheimer's disease: a clinicopathologic study of 57 cases. Neurology. 1988;38:359-64.

20. Shaw LM, Vanderstichele $H$, Knapik-Czajka M, Clark CM, Aisen PS, Petersen RC, Blennow K, Soares $H$, Simon A, Lewczuk P, Dean R, Siemers E, Potter W, Lee VM, Trojanowski JQ. Cerebrospinal fluid biomarker signature in Alzheimer's disease neuroimaging initiative subjects. Ann Neurol. 2009;65:403-13.

21. Risacher SL, Saykin AJ. Neuroimaging and other biomarkers for Alzheimer's disease: the changing landscape of early detection. Annu Rev Clin Psychol. 2013;9:621-48.

22. Arendt T. Synaptic degeneration in Alzheimer's disease. Acta Neuropathol. 2009:118:167-79.

23. Bark IC, Hahn KM, Ryabinin AE, Wilson MC. Differential expression of SNAP25 protein isoforms during divergent vesicle fusion events of neural development. Proc Natl Acad Sci U S A. 1995:92:1510-4.

24. Walch-Solimena C, Blasi J, Edelmann L, Chapman ER, von Mollard GF, Jahn $\mathrm{R}$. The t-SNAREs syntaxin 1 and SNAP-25 are present on organelles that participate in synaptic vesicle recycling. J Cell Biol. 1995;128:637-45.

25. Hou QL, Gao X, Lu Q, Zhang XH, Tu YY, Jin ML, Zhao GP, Yu L, Jing NH, Li BM. SNAP-25 in hippocampal CA3 region is required for long-term memory formation. Biochem Biophys Res Commun. 2006:347:955-62.

26. Davidsson P, Puchades M, Blennow K. Identification of synaptic vesicle, preand postsynaptic proteins in human cerebrospinal fluid using liquid-phase isoelectric focusing. Electrophoresis. 1999:20:431-7.

27. Mattsson N, Insel PS, Palmqvist S, Portelius E, Zetterberg H, Weiner M Blennow K, Hansson O. Cerebrospinal fluid tau, neurogranin, and neurofilament light in Alzheimer's disease. EMBO Mol Med. 2016;8:1184-96.

28. Salomone S, Caraci F, Leggio GM, Fedotova J, Drago F. New pharmacological strategies for treatment of Alzheimer's disease: focus on disease modifying drugs. Br J Clin Pharmacol. 2012;73:504-17.

Ready to submit your research? Choose BMC and benefit from:

- fast, convenient online submission

- thorough peer review by experienced researchers in your field

- rapid publication on acceptance

- support for research data, including large and complex data types

- gold Open Access which fosters wider collaboration and increased citations

- maximum visibility for your research: over $100 \mathrm{M}$ website views per year

At $\mathrm{BMC}$, research is always in progress.

Learn more biomedcentral.com/submissions 